\title{
NUMERICAL SPECTRAL LEGENDRE APPROACH FOR SOLVING SPACE-TIME FRACTIONAL ADVECTION-DISPERSION PROBLEMS
}

\author{
E.H. Doha* W.M. Abd-Elhameed ${ }^{\dagger}$ N.A. Elkot $\ddagger$ and Y.H. Youssri ${ }^{\S}$ \\ Department of Mathematics, Faculty of Science, Cairo University, Giza 12613, Egypt
}

Received 21/6/2017 $\quad$ Revised 28/8/20017 Accepted 27/11/2017

\begin{abstract}
This manuscript is devoted to implementing spectral numerical solutions to two kinds of fractional space-time advectiondispersion problems governed by certain constraints conditions. The collocation and tau spectral methods are utilized for obtaining the proposed spectral solutions. A double Legendre expansion is proposed as an approximate solution. The main idea of the algorithm is basically depend on converting the equation with its constraints conditions into linear or nonlinear systems of algebraic equations which can be efficiently solved with the aid of suitable numerical solvers. Some illustrative examples are displayed aiming to confirm robustness, efficiency and accuracy of the proposed spectral solutions.
\end{abstract}

MSC: $65 \mathrm{M} 70,34 \mathrm{~A} 08,33 \mathrm{C} 45$

Keywords. Legendre polynomials; spectral methods; advection-dispersion problems; fractional differential equations

\section{Introduction}

The importance of the fractional calculus has greatly increased due to the numerous applications in different disciplines. In fact, a variety of important problems in physics, biology, chemistry, control theory, signal processing, fluid mechanics and some other branches can be modeled by fractional differential equations (FDEs) or fractional partial differential equations (FPDEs). As an example, when describing anomalous diffusion, such as contaminants transport in the soil, oil flow in porous media, groundwater flow and turbulence, there is an evidence that fractional models are efficient to capture some important features of particles transport, such as particles with velocity variation and long-rest periods. This is due to the nonlocal property of the fractional operator. The majority of FDEs and FPDEs do not have analytical solutions, this of course gives the numerical investigations for these kinds of equations a great importance.

There are three kinds of FPDEs. They are space, time and space-time FPDEs. Among the essential FDEs is the fractional advection-diffusion equation. Numerous studies are performed for obtaining numerical solutions to solve it. In this regard, the authors in [1] studied the stability of finite difference methods for solving space-time fractional advection-diffusion equation (STFADE), Zhuang et al. [2], suggested a numerical algorithm to solve STFADE with nonlinear source term, Jiang et al. [3] derived some analytical solutions for the multi-term time-space Caputo-Riesz fractional advection-diffusion equations on a finite domain. Adomian decomposition method is utilized in [4] to solve an intermediate fractional advection-dispersion equation. The authors in [5] derived the fundamental solution for the space-time Riesz-Caputo fractional advection-diffusion equation with an initial condition. In [6], the authors suggested a spectral representation of the fractional Laplacian operator and used the equivalent relationship between fractional Laplacian operator and Riesz fractional derivative. In addition

\footnotetext{
*Correspondence: eiddoha@sci.cu.edu.eg

†waleed@sci.cu.edu.eg

$\ddagger$ anermeen@sci.cu.edu.eg

$\S$ youssri@sci.cu.edu.eg
} 
they derived analytical solution for the multi-term, time-space, Caputo-Riesz fractional advection-diffusion equation on a finite domain. The authors in [7] employed a finite difference method to solve space fractional transient advection-diffusion equation, while the authors in [8] utilized a finite element method to solve the fractional advection-dispersion equation. Zheng et al. [9] developed another finite element method to solve the space fractional advection-diffusion equation with non-homogeneous initial-boundary conditions. The homotopy perturbation method is also employed in [10] to treat the fractional advection-dispersion equation. Some other techniques for handling this equation can be found in [11-24]).

Utilizing the different versions of spectral methods has greatly increased. These methods are used for numerically solving almost all types of differential equations. In spectral methods, it is assumed that the proposed spectral solution is expressed as a linear combination of certain basis functions which are often orthogonal. Many articles that consider orthogonal polynomials as basis functions for solving different kinds of differential equations. It is well-known that there are three celebrated types of spectral methods, namely, Galerkin, tau, and collocation methods. Each type has its own role in approximation. Galerkin method is extensively used for treating linear boundary and initial value problems (see, for instance $[25,26])$. The tau method is useful when we treat differential equations with complicated constraints (see, for example [27]). Collocation method is employed in a wide class of differential equations due to its efficiency, and in particular, in treating nonlinear problems. There are numerous articles employed this method (see, for example [28, 29]).

The principal aim of the current paper is to obtain numerical solutions for two kinds of fractional advection dispersion equations (FADEs). The two spectral methods, namely, tau and collocation methods are employed. The application of the two proposed methods reduces the solution of the equation with its boundary and initial conditions into a system of algebraic equations which can be solved by utilizing suitable numerical solvers.

The materials of the paper are as follows. Section 2 is interested in presenting some elementary facts of the fractional calculus. Further, some properties of shifted Legendre polynomials are presented in this section. Section 3 is interested in solving the first kind of fractional advection-dispersion equation using two different spectral techniques. The same spectral methods are used for solving the second kind of fractional advection-dispersion equation in Section 4 . Section 5 gives a comprehensive study on convergence and error of the proposed expansion. Some numerical experiments are displayed in Section

6 to validate the applicability and efficiency of the proposed numerical methods. Finally, some conclusions are reported in Section 7 .

\section{Preliminaries and used formulae}

In this section, some fundamentals of fractional calculus theory are displayed. In addition, some basic properties and formulae concerned with shifted Legendre polynomials are also given.

\subsection{Some facts of fractional calculus}

Definition 1. Let $I^{\eta}$ denote the Riemann-Liouville fractional integral operator of order $\eta$ on the usual Lebesgue space $L_{1}[0,1]$, then $I^{\eta}$ is defined as:

$$
I^{\eta} f(z)= \begin{cases}\frac{1}{\Gamma(\eta)} \int_{0}^{z}(z-\tau)^{\eta-1} f(\tau) d \tau, & \eta>0, \\ f(z), & \eta=0 .\end{cases}
$$

This operator satisfies the following properties:

$$
\begin{aligned}
& \text { (i) } I^{\eta} I^{\xi}=I^{\eta+\xi} \\
& \text { (ii) } I^{\eta} I^{\xi}=I^{\eta} I^{\xi} \\
& \text { (iii) } I^{\eta} t^{\theta}=\frac{\Gamma(\theta+1)}{\Gamma(\theta+\eta+1)} t^{\eta+\theta}
\end{aligned}
$$

where $\eta, \xi \geqslant 0$, and $\theta>-1$. 
Definition 2. Let ${ }_{a}^{R} D_{z}^{\xi}$ and ${ }_{z}^{R} D_{b}^{\xi} f$ denote, respectively, the left and right handed Riemann-Liouville fractional-order of derivatives, then they are defined as:

$$
\begin{aligned}
& \left({ }_{a}^{R} D_{z}^{\xi} f\right)(z)=\frac{1}{\Gamma(\ell-\xi)} \frac{d^{\ell}}{d z^{\ell}} \int_{a}^{z}(z-\tau)^{\ell-\xi-1} f(\tau) d \tau, \\
& \left({ }_{z}^{R} D_{b}^{\xi} f\right)(z)=\frac{(-1)^{\ell}}{\Gamma(\ell-\xi)} \frac{d^{\ell}}{d z^{\ell}} \int_{z}^{b}(\tau-z)^{\ell-\xi-1} f(\tau) d \tau .
\end{aligned}
$$

Definition 3. Let ${ }_{a}^{C} D_{z}^{\xi}$ and ${ }_{z}^{C} D_{b}^{\xi} f$ denote, respectively, the left and right handed Caputo fractional-order of derivatives, then they are defined as:

$$
\begin{aligned}
& \left({ }_{a}^{C} D_{z}^{\xi} f\right)(z)=\frac{1}{\Gamma(\ell-\xi)} \int_{a}^{z}(z-\tau)^{\ell-\xi-1} f^{(\ell)}(\tau) d \tau, \xi>0, t>0, \\
& \left({ }_{z}^{C} D_{b}^{\xi} f\right)(z)=\frac{(-1)^{\ell}}{\Gamma(\ell-\xi)} \int_{z}^{b}(\tau-z)^{\ell-\xi-1} f^{(\ell)}(\tau) d \tau, \xi>0, t>0,
\end{aligned}
$$

where $\ell-1 \leqslant \xi<\ell, \ell \in \mathbb{N}$.

The following properties are satisfied by the operator $D^{\xi}$ for $\ell-1 \leqslant \alpha<\ell$,

$$
\begin{aligned}
\left(D^{\xi} I^{\xi} f\right)(z) & =f(t), \\
\left(I^{\xi} D^{\xi} f\right)(z) & =f(z)-\sum_{j=0}^{\ell-1} \frac{f^{(j)}\left(0^{+}\right)}{j !}(z-a)^{j}, z>0, \\
D^{\xi} z^{j} & =\frac{\Gamma(j+1)}{\Gamma(j+1-\xi)} z^{j-\xi}, \quad j \in \mathbb{N}, j \geq\lceil\xi\rceil .
\end{aligned}
$$

Definition 4. The Riesz fractional-order derivative is defined as (see, [2, 5, 30])

$$
\frac{\partial^{\xi} f}{\partial|z|^{\xi}}=-c\left[-\infty^{R} D_{z}^{\xi} f(z)+_{z}^{R} D_{\infty}^{\xi} f(z)\right]
$$

where $c=\frac{1}{2} \sec \frac{\xi \pi}{2}$. If $f$ is defined in the interval $[a, b]$, with $f(a)=f(b)=0$, then it can be assumed that $f(z)=0$ for all $z>a$ and $z<b$. Therefore, we have

$$
\frac{\partial^{\xi} f(z)}{\partial|z|^{\xi}}=-c\left[{ }_{a}^{R} D_{z}^{\xi} f(z)+{ }_{z}^{R} D_{b}^{\xi} f(z)\right]
$$

For additional properties of fractional derivatives and integrals, one can consult [31,32].

\subsection{Relevant properties and formulae of shifted Legendre polynomials}

The standard Legendre polynomials $\left\{P_{k}(z): k=0,1, \ldots\right\} ; z \in[-1,1]$ form a complete orthogonal system for $L^{2}(-1,1)$. Furthermore, the set $\left\{P_{k}(x): k=0,1, \ldots\right\}$ is orthogonal on $[-1,1]$ due to the relation

$$
\int_{-1}^{1} P_{m}(z) P_{n}(z) d z=\frac{2 \delta_{m n}}{2 n+1}
$$

where $\delta_{m n}$ is the celebrated Kronecker delta function.

Rodrigues' formula of Legendre polynomials is

$$
P_{n}(z)=\frac{1}{2^{n} n !} \frac{d^{n}}{d z^{n}}\left(z^{2}-1\right)^{n} .
$$

We denote by $P_{k}^{\ell}(z)$ the shifted Legendre polynomials defined on $(0, \ell)$ as:

$$
P_{k}^{\ell}(z)=P_{k}\left(\frac{2 z}{\ell}-1\right) \text {. }
$$


The following two analytic forms of shifted Legendre polynomials are of important use in the sequel (see, $[33,34]$ )

$$
\begin{gathered}
P_{k}^{\ell}(z)=\sum_{i=0}^{k} \frac{(-1)^{k+i}(k+i) !}{(k-i) !(i !)^{2} \ell^{i}} z^{i}, \\
P_{k}^{\ell}(z)=\sum_{i=0}^{k} \frac{(k+i) !}{(i !)^{2}(k-i) ! \ell^{i}}(z-\ell)^{i} .
\end{gathered}
$$

For any function $f(z) \in L^{2}[0, \ell]$, assume that it has the following expansion

$$
f(z)=\sum_{k=0}^{\infty} c_{k} P_{k}^{\ell}(z)
$$

where

$$
c_{k}=\frac{2 k+1}{\ell} \int_{0}^{\ell} f(z) P_{k}^{\ell}(z) d z .
$$

Consider the following approximation of $f(z)$

$$
f(z) \approx f_{m}(z)=\sum_{k=0}^{m} c_{k} P_{k}^{\ell}(z) .
$$

The following two integral formulae are useful in what follows.

Lemma 1. For every nonnegative integer $r$ and any real number $k$, one has

$$
\int_{0}^{\ell} z^{k} P_{r}^{\ell}(z) d z=\frac{\ell^{k+1}(\Gamma(k+1))^{2}}{\Gamma(k-r+1) \Gamma(k+r+2)} .
$$

Proof. If we make use of the analytic form (2.9), then we get

$$
\int_{0}^{\ell} z^{k} P_{r}^{\ell}(z) d z=\ell^{k+1} \sum_{j=0}^{r} \frac{(-1)^{r+j}(r+j) !}{(j !)^{2}(r-j) !(j+k+1)} .
$$

Now, if we set

$$
S_{k, r}=\sum_{j=0}^{r} \frac{(-1)^{r+j}(r+j) !}{(j !)^{2}(r-j) !(j+k+1)},
$$

then with the aid of Zeilberger's algorithm [35], it can be shown that $S_{k, r}$ satisfies the following difference equation of order one:

$$
(r-k) S_{k, r}+(r+k+2) S_{k, r+1}=0, \quad S_{k, 0}=\frac{1}{k+1} .
$$

The above recurrence relation can be easily solved to give

$$
S_{k, r}=\frac{\ell^{k+1}\left(\Gamma(k+1)^{2}\right.}{\Gamma(k-r+1) \Gamma(k+r+2)} .
$$

This proves formulae (2.14).

The following Beta integral formula is needed in the sequel.

Lemma 2.

$$
\int_{0}^{\ell} z^{m}(\ell-z)^{\beta} d z=\ell^{m+\beta+1} \frac{\Gamma(m+1) \Gamma(\beta+1)}{\Gamma(m+\beta+2)} .
$$

Proof. Using the substitution $z=\ell t$, we have

$$
\int_{0}^{\ell} z^{m}(\ell-z)^{\beta} d z=\ell^{m+\beta+1} \int_{0}^{1} t^{m}(1-t)^{\beta} d t=\ell^{m+\beta+1} \frac{\Gamma(m+1) \Gamma(\beta+1)}{\Gamma(m+\beta+2)} .
$$




\section{The first kind of the space-time fractional advection-dispersion equations}

The main objective of the current section is to derive in detail two spectral algorithms for the numerical solution of the first kind of the space-time fractional linear advection-dispersion problems.

For our present purposes, we define the following operator

$$
\mathcal{B}_{i} y(z, t)=\left(\frac{\partial}{\partial z}+i\right) y(z, t) .
$$

Now, consider the following space-time Riemann-Liouville fractional advection diffusion equation (see, [36]):

$$
\begin{aligned}
& { }^{C} D_{t}^{\alpha} y(z, t)-a(z)^{R} D_{z}^{\beta} y(z, t)+b(z){ }^{R} D_{z}^{\gamma} y(z, t)=f(z, t) \\
& 1<\alpha \leq 2, \quad 1 \leq \beta<2, \quad 0<\gamma \leq 1, \quad(z, t) \in \Omega:=(0, \ell) \times(0, \tau),
\end{aligned}
$$

subject to the boundary conditions:

$$
\mathcal{B}_{s} y(0, t)=\mu_{1}(t), \quad \mathcal{B}_{k} y(\ell, t)=\mu_{2}(t) ; \quad 0<t<\tau
$$

and the initial conditions:

$$
y(z, 0)=f_{0}(z) \quad \text { and } \quad y_{t}(z, 0)=h_{0}(z) ; \quad 0<z<\ell,
$$

where $y(z, t)$ represents the concentration, $a(z)>0, b(z)>0, \mu_{1}(t), \mu_{2}(t), f_{0}(z), h_{0}(z)$ are given continuous functions, $s, k$ are given constants and $f(z, t)$ is the source term.

\subsection{Double expansion selection}

Consider the two families of shifted Legendre polynomials

$$
\phi_{i}(z)=P_{i}^{\ell}(z), i=0,1,2, \ldots,
$$

and

$$
\psi_{j}(t)=P_{j}^{\tau}(t), j=0,1,2, \ldots
$$

We observe that $\left\{\phi_{i}(z)\right\}_{i \geq 0}$ is an orthogonal linearly independent set on $[0, \ell]$ with respect to $w(z)=1$. In fact

$$
\int_{0}^{\ell} \phi_{i}(z) \phi_{j}(z) d z=h_{i, j}= \begin{cases}\frac{\ell}{2 i+1}, & i=j \\ 0, & i \neq j\end{cases}
$$

Consider a function $y(z, t)$ which can be expanded as

$$
y(z, t)=\sum_{i=0}^{\infty} \sum_{j=0}^{\infty} c_{i j} \phi_{i}(z) \psi_{j}(t)
$$

where

$$
c_{i j}=\frac{(2 i+1)(2 j+1)}{\ell \tau} \int_{0}^{\tau} \int_{0}^{\ell} y(z, t) \phi_{i}(z) \psi_{j}(t) d t .
$$

The following two theorems are useful in what follows.

Theorem 1. In Riemann-Liouville sense, the following fractional derivative relation holds for $\alpha \in(1,2)$

$$
{ }^{R} D^{\alpha} \phi_{i}(z)=\sum_{k=0}^{i} \frac{(-1)^{i+k}(k+i) ! \Gamma(\alpha-k) \sin (\pi(\alpha-k))}{\pi(i-k) ! k ! \ell^{k}} z^{k-\alpha} .
$$


Proof. Due to the power form representation of $P_{i}^{\ell}(z)$ in (2.9), one can write

$$
\phi_{j}(z)=\sum_{r=0}^{j} \frac{(-1)^{j-r}(j+r) !}{(r !)^{2}(j-r) ! \ell^{r}} z^{r} .
$$

If the operator ${ }^{R} D^{\alpha}$ is applied to both sides of (3.9), then formula (2.6) yields

$$
{ }^{R} D^{\alpha} \phi_{j}(z)=\sum_{r=0}^{j} \frac{(-1)^{j-r}(j+r) !}{(j-r) ! r ! \Gamma(r+1-\alpha) \ell^{r}} z^{r-\alpha} .
$$

Now, if we use the identity

$$
\Gamma(\xi) \Gamma(1-\xi)=\frac{\pi}{\sin (\xi \pi)},
$$

and often performing some computations, then formula (3.8) is obtained.

Similarly, we can prove the following theorem.

Theorem 2. In Caputo sense, the following fractional derivative relation holds for $\alpha \in(1,2)$

$$
{ }^{C} D^{\alpha} \psi_{j}(t)=\sum_{s=\lceil\beta\rceil}^{j} \frac{(-1)^{j+s} \Gamma(\alpha-s) \sin (\pi(\alpha-s))}{\pi(j-s) ! s ! \tau^{s}} t^{s-\alpha} .
$$

\subsection{The collocation approach for treating Eq. (3.1)}

We consider an approximate numerical solution of Eq. (3.1) written as

$$
y_{N}(z, t)=\sum_{i=0}^{N} \sum_{j=0}^{N} c_{i j} \phi_{i}(z) \psi_{j}(t)
$$

and consider the following approximations for $a(z)$ and $b(z)$ in the forms

$$
\begin{aligned}
& a(z) \approx \sum_{p=0}^{N} a_{p} P_{p}^{\ell}(z), \\
& b(z) \approx \sum_{q=0}^{N} b_{q} P_{q}^{\ell}(z) .
\end{aligned}
$$

Now, the residual $R(z, t)$ of $(3.1)$ is

$$
\begin{aligned}
& R(z, t)=\sum_{i=0}^{N} \sum_{j=0}^{N} c_{i j} P_{i}^{\ell}(z){ }^{C} D_{t}^{\alpha} \psi_{j}(t)-a(z) \sum_{i=0}^{N} \sum_{j=0}^{N} c_{i j} P_{j}^{\tau}(t){ }^{R} D_{z}^{\beta} P_{i}^{\ell}(z) \\
& +b(z) \sum_{i=0}^{N} \sum_{j=0}^{N} c_{i j} P_{j}^{\tau}(t){ }^{R} D_{z}^{\gamma} P_{i}^{\ell}(z)-f(z, t) .
\end{aligned}
$$

With the aid of the two analytic forms of $\phi_{i}(z)$ and $\psi_{j}(t)$, and the two formulae (3.8) and (3.11), the residual $R(z, t)$ of (3.13) can be turned into

$$
\begin{aligned}
R(z, t) & =\sum_{i=0}^{N} \sum_{j=0}^{N} \sum_{s=\lceil\alpha\rceil}^{j} c_{i j} P_{i}^{\ell}(z) \frac{(-1)^{j-s}(j+s) !(\alpha-s-1) ! \sin (\pi(\alpha-s))}{\pi(j-s) ! s ! \tau^{s}} t^{s-\alpha} \\
& -\sum_{p=0}^{N} \sum_{i=0}^{N} \sum_{j=0}^{N} \sum_{k=0}^{i} a_{p} c_{i j} P_{j}^{\tau}(t) P_{p}^{\ell}(z) \frac{(-1)^{i+k}(i+k) !(\beta-k-1) ! \sin (\pi(\beta-k))}{\pi(i-k) ! k ! \ell^{k}} z^{k-\beta} \\
& +\sum_{q=0}^{N} \sum_{i=0}^{N} \sum_{j=0}^{N} \sum_{k=0}^{i} b_{q} c_{i j} P_{j}^{\tau}(t) P_{q}^{\ell}(z) \frac{(-1)^{i+k}(i+k) !(\gamma-k-1) ! \sin (\pi(\gamma-k))}{\pi(i-k) ! k ! \ell^{k}} z^{k-\gamma}-f(z, t),
\end{aligned}
$$


To apply the typical collocation method on Eq. (3.1), we enforce the residual given in (3.14) to vanish at some suitable nodes. The set of nodes is taken as: $\left\{\left(\frac{i \ell}{N+1}, \frac{j \tau}{N+1}\right): 1 \leq i, j \leq N\right\}$. In such case, we have

$$
R\left(z_{i}, t_{j}\right)=0, \quad 1 \leq i, j \leq N,
$$

moreover, the boundary and initial conditions (3.1) and (3.3) yield

$$
\begin{gathered}
\mathcal{B}_{s} y\left(0, t_{j}\right)=\mu_{1}\left(t_{j}\right), \quad 1 \leq j \leq\left\lfloor\frac{N}{2}\right\rfloor, \\
\mathcal{B}_{k} y\left(\ell, t_{j}\right)=\mu_{2}\left(t_{j}\right), \quad 1 \leq j \leq\left\lfloor\frac{N}{2}\right\rfloor, \\
y\left(z_{i}, 0\right)=f_{0}\left(z_{i}\right), \quad 1 \leq i \leq\left\lfloor\frac{N}{2}\right\rfloor+1, \\
y_{t}\left(z_{i}, 0\right)=h_{0}\left(z_{i}\right), \quad 1 \leq i \leq\left\lfloor\frac{N}{2}\right\rfloor+\varrho,
\end{gathered}
$$

where

$$
\varrho= \begin{cases}2, & \mathrm{~N} \text { is odd } \\ 0, & \mathrm{~N} \text { is even. }\end{cases}
$$

Therefore, an algebraic system of equations whose dimension is $(N+1)^{2}$ in the vector of unknowns $\mathbf{C}=\left\{c_{i j}: 0 \leq i, j \leq N\right\}$ is generated. It can be solved by a suitable solver. Hence, the desired approximate solution can be obtained.

\subsection{The tau approach for handling Eq. (3.1)}

This section is interested in introducing a tau algorithm for handling spectral approximate solution to Eq. (3.1). The application of tau method leads to

$$
\int_{0}^{\tau} \int_{0}^{\ell} R(z, t) \rho_{i j}(z, t) d z d t=0, \quad 1 \leq i, j \leq N+1
$$

where $\rho_{i j}(z, t)$ is given by

$$
\rho_{i j}(z, t)=P_{i}^{\ell}(z) P_{j}^{\tau}(t),
$$

and $R(z, t)$ is as given in (3.14).

Now, Eq. (3.20) can be written alternatively in the form

$$
\begin{aligned}
& \sum_{i=0}^{N} \sum_{j=0}^{N} \sum_{s=\lceil\alpha\rceil}^{j} \sum_{k=0}^{i} c_{i j} \frac{(-1)^{i+k+j-s}(j+s) !(i+k) !(\alpha-s-1) ! \sin (\pi(\alpha-s))}{\pi(j-s) !(i-k) ! s !(k !)^{2} \ell^{k} \tau^{s}} \int_{0}^{\ell} z^{k} P_{m}^{\ell}(z) d z \int_{0}^{\tau} t^{s-\alpha} P_{n}^{\tau}(t) d t \\
& -\sum_{i=0}^{N} \sum_{j=0}^{N} \sum_{k=0}^{i} \sum_{s=0}^{j} \sum_{r=0}^{p} a_{p} c_{i j} \frac{(-1)^{i+k+j-s+r+p}(\beta-k-1) !(j+s) !(i+k) !(r+p) ! \sin (\pi(\beta-k))}{\pi(i-k) !(j-s) !(p-r) !(s !)^{2}(r !)^{2} k ! \ell^{r+k} \tau^{s}} \\
& \times \int_{0}^{\ell} z^{r+k-\beta} P_{m}^{\ell}(z) d z \int_{0}^{\tau} t^{s} P_{n}^{\tau}(t) d t \\
& -\sum_{i=0}^{N} \sum_{j=0}^{N} \sum_{k=0}^{i} \sum_{s=0}^{j} \sum_{l=0}^{q} b_{q} c_{i j} \frac{(-1)^{i+k+j-s+l+q}(\gamma-k-1) !(j+s) !(i+k) !(l+q) ! \sin (\pi(\gamma-k))}{\pi(i-k) !(j-s) !(q-l) ! k !(s !)^{2}(l !)^{2} \ell^{l+k} \tau^{s}} \\
& \times \int_{0}^{\ell} z^{l+k-\gamma} P_{m}^{\ell}(z) d z \int_{0}^{\tau} t^{s} P_{n}^{\tau}(t) d t-f_{m n}=0 .
\end{aligned}
$$


Making use of Lemma 1, then Eq. (3.21) can be also written alternatively as:

$$
\begin{aligned}
& \sum_{i=0}^{N} \sum_{j=0}^{N} \sum_{s=\lceil\alpha\rceil}^{j} \sum_{k=0}^{i} c_{i j} \frac{(-1)^{i+k+j-s}(j+s) !(i+k) !(k !)^{2}((s-\alpha) !)^{2}(\alpha-s-1) ! \sin (\pi(\alpha-s)) \ell \tau^{-\alpha+1}}{\pi(j-s) !(i-k) !(k-m) !(k+N+1) !(s-\alpha-n) !(s-\alpha+n+1) ! s !(k !)^{2}} \\
& -\sum_{i=0}^{N} \sum_{j=0}^{N} \sum_{k=0}^{i} \sum_{s=0}^{j} \sum_{r=0}^{p} a_{p} c_{i j} \frac{(-1)^{i+k+j-s+r+p}(\beta-k-1) !(j+s) !(i+k) !(r+p) !((r+k-\beta) !)^{2} \sin (\pi(\beta-k)) \ell^{-\beta+1} \tau}{\pi(i-k) !(j-s) !(p-r) !(r !)^{2}(r+k-\beta-m) !(r+k-\beta+N+1) !(s-n) !(s+n+1) ! k !} \\
& -\sum_{i=0}^{N} \sum_{j=0}^{N} \sum_{k=0}^{i} \sum_{s=0}^{j} \sum_{l=0}^{q} b_{q} c_{i j} \frac{(-1)^{i+k+j-s+l+q}(\gamma-k-1) !(j+s) !(i+k) !(l+q) !((l+k-\gamma) !)^{2} \sin (\pi(\gamma-k)) \ell^{-\gamma+1} \tau}{\pi(i-k) !(j-s) !(q-l) ! k !(l+k-\gamma-m) !(l+k-\gamma+N+1) !(s-n) !(s+n+1) !(l !)^{2}} \\
& -f_{m n}=0, \quad 1 \leq m, n \leq N,
\end{aligned}
$$

Also, the boundary and initial conditions $(3.2),(3.3)$ yield

$$
\begin{gathered}
\mathcal{B}_{s} y\left(0, t_{j}\right)=\mu_{1}\left(t_{j}\right), \quad 1 \leq j \leq\left\lfloor\frac{N}{2}\right\rfloor, \\
\mathcal{B}_{k} y\left(\ell, t_{j}\right)=\mu_{2}\left(t_{j}\right), \quad 1 \leq j \leq\left\lfloor\frac{N}{2}\right\rfloor, \\
y\left(z_{i}, 0\right)=f_{0}\left(z_{i}\right), \quad 1 \leq i \leq\left\lfloor\frac{N}{2}\right\rfloor+1, \\
y_{t}\left(z_{i}, 0\right)=h_{0}\left(z_{i}\right), \quad 1 \leq i \leq\left\lfloor\frac{N}{2}\right\rfloor+\varrho,
\end{gathered}
$$

where $\varrho= \begin{cases}2, & \mathrm{~N} \text { is odd; } \\ 0, & \mathrm{~N} \text { is even. }\end{cases}$

Eqs. (3.22)-(3.26) generate an algebraic system of equations of dimension $(N+1)^{2}$ in the unknowns $c_{i j}$ which can be efficiently solved.

\section{The second kind of space-time fractional advection-dispersion equations}

This section focuses on solving the second kind of space-time fractional linear advection-dispersion problems. The two spectral methods used in Section 3 are also used in this section for obtaining the proposed numerical solutions.

Consider the space-time Riemann-Liouville fractional advection-dispersion problem with the Riesz space fractional derivatives(see, [6]):

$$
\begin{aligned}
& \left({ }^{R} D_{t}^{\alpha} y\right)(z, t)-\kappa_{\beta}\left({ }^{R} D_{|z|}^{\beta} y\right)(z, t)-\kappa_{\gamma}\left({ }^{R} D_{|z|}^{\gamma} y\right)(z, t)=f(z, t) \\
& 0<\alpha \leq 1, \quad 0<\beta<1, \quad 1<\gamma<2, \quad(z, t) \in \Omega:=(0, \ell) \times(0, \tau),
\end{aligned}
$$

governed by the following boundary-initial conditions:

$$
\mathcal{B}_{s} y(0, t)=\mu_{1}(t), \quad \mathcal{B}_{k} y(\ell, t)=\mu_{2}(t) ; \quad 0<t<\tau,
$$

and

$$
y(z, 0)=f_{0}(z) ; \quad 0<z<\ell,
$$

where $y(z, t)$ is the concentration, $\kappa_{\beta}$ and $\kappa_{\gamma}$ are, respectively, the dispersion coefficient and the average fluid velocity, and $f(z, t)$ is the source term.

First, the following theorem is useful in what follows. 
Theorem 3. The following Riesz fractional derivative relation holds for $\beta \in(0,2)-\{1\}$

$$
\frac{\partial^{\beta} \phi_{i}(z)}{\partial|z|^{\beta}}=\sum_{k=0}^{i} \frac{(-1)^{k+1}(i+k) ! \Gamma(\beta-k) \sec \left(\frac{\pi \beta}{2}\right) \sin (\pi(\beta-k))}{2 \pi(i-k) ! k ! \ell^{k}}\left((-1)^{i} z^{k-\beta}+(\ell-z)^{k-\beta}\right) .
$$

Proof. If we make use of the power form representations of $P_{i}^{\ell}(z)$ namely, $(2.9),(2.10)$, then after performing some computations similar to those given in the proof of Theorem 1, relation (4.4) can be obtained.

\subsection{A collocation approach for handling Eq. (4.1)}

Now, assume an approximate solution of Eq. (4.1) written as in (3.12). The residual of (4.1) is

$$
\begin{aligned}
R(z, t) & =\sum_{i=0}^{N} \sum_{j=0}^{N} c_{i j} P_{i}^{\ell}(z)^{C} D_{t}^{\alpha} \psi_{j}(t)-\kappa_{\beta} \sum_{i=0}^{N} \sum_{j=0}^{N} c_{i j} P_{j}^{\tau}(t)^{R} D_{|z|}^{\beta} \phi_{i}(z) \\
& -\kappa_{\gamma} \sum_{i=0}^{N} \sum_{j=0}^{N} c_{i j} P_{j}^{\tau}(t)^{R} D_{|z|}^{\beta} \phi_{i}(z)-f(z, t) .
\end{aligned}
$$

If we make use of formula (4.5) together with the two analytic formulae of $\phi_{i}(z)$ and $\psi_{j}(t)$, and formulae $(3.11)$ and $(4.4)$, then the residual $R(z, t)$ can be written as

$$
\begin{aligned}
R(z, t) & =\sum_{i=0}^{N} \sum_{j=0}^{N} \sum_{s=\lceil\alpha\rceil}^{j} \sum_{k=0}^{i} c_{i j} \frac{(-1)^{j+s+k+1}(k+i) ! \Gamma(\alpha-s) \sin (\pi(\alpha-s))}{\pi(i-k) !(k !)^{2}(j-s) ! s ! \ell^{k} \tau^{s}} t^{s-\alpha} z^{k} \\
& -\kappa_{\beta} \sum_{i=0}^{N} \sum_{j=0}^{N} \sum_{k=0}^{i} \sum_{s=0}^{j} c_{i j} \frac{(-1)^{s+j+k+1}(s+j) !(i+k) ! \Gamma(\beta-k) \sec \left(\frac{\pi \beta}{2}\right) \sin (\pi(\beta-k))}{2 \pi(j-s) !(s !)^{2}(i-k) ! k ! \ell^{k} \tau^{s}}\left((-1)^{i} z^{k-\beta}+(\ell-z)^{k-\beta}\right) t^{s} \\
& -\kappa_{\gamma} \sum_{i=0}^{N} \sum_{j=0}^{N} \sum_{k=0}^{i} \sum_{s=0}^{j} c_{i j} \frac{(-1)^{s+j+k+1}(s+j)(i+k) ! \Gamma(\gamma-k) \sec \left(\frac{\pi \gamma}{2}\right) \sin (\pi(\gamma-k))}{2 \pi(j-s) !(s !)^{2}(i-k) ! k ! \ell^{k} \tau^{s}}\left((-1)^{i} z^{k-\gamma}+(\ell-z)^{k-\gamma}\right) t^{s} \\
& -f(z, t) .
\end{aligned}
$$

Now, if we choose the set of nodes $\left\{\left(\frac{i \ell}{N+1}, \frac{j \tau}{N+1}\right): 1 \leq i, j \leq N\right\}$, and apply collocation method on Eq. (4.6), then we get

$$
R\left(z_{i}, t_{j}\right)=0,1 \leq i, j \leq N
$$

Moreover, the conditions (4.2) and (4.3) yield the equations

$$
\begin{gathered}
\mathcal{B}_{s} y\left(0, t_{j}\right)=\mu_{1}\left(t_{j}\right), \quad 1 \leq j \leq\left\lfloor\frac{N}{2}\right\rfloor, \\
\mathcal{B}_{k} y\left(\ell, t_{j}\right)=\mu_{2}\left(t_{j}\right), \quad 1 \leq j \leq\left\lfloor\frac{N}{2}\right\rfloor+1, \\
y\left(z_{i}, 0\right)=f_{0}\left(z_{i}\right), \quad 1 \leq i \leq N+\rho,
\end{gathered}
$$

where $\rho= \begin{cases}0, & \mathrm{~N} \text { even; } \\ 1, & \mathrm{~N} \text { odd. }\end{cases}$

Eqs. (4.7)-(4.10) generate an algebraic system of equations of dimension $(N+1)^{2}$ in the vector of unknowns $\mathbf{C}=\left\{c_{i j}: 0 \leq\right.$ $i, j \leq N\}$. Hence, the approximate spectral solution can be obtained. 


\subsection{The tau approach for handling Eq. (4.1)}

If we apply the tau method to Eq. (4.1), and if we select the test functions as:

$$
\rho_{i j}(z, t)=P_{i}^{\ell}(z) P_{j}^{\tau}(t)
$$

then we get

$$
\int_{0}^{\tau} \int_{0}^{\ell} R(z, t) \rho_{i j}(z, t) d z d t=0, \quad 1 \leq i, j \leq N+1
$$

where $R(z, t)$ is given as in (4.6). Now, Eq. (4.11) may be written in the following alternative form

$$
\begin{aligned}
& \sum_{i=0}^{N} \sum_{j=0}^{N} \sum_{s=\lceil\alpha\rceil}^{j} \sum_{k=0}^{i} c_{i j} \frac{(-1)^{j+s+k+1}(k+i) ! \Gamma(\alpha-s) \sin (\pi(\alpha-s))}{\pi(i-k) !(k !)^{2}(j-s) ! s ! \ell^{k} \tau^{s}} \int_{0}^{\ell} z^{k} P_{m}^{\ell}(z) d z \int_{0}^{\tau} t^{s-\alpha} P_{n}^{\tau}(t) d t \\
& -\kappa_{\beta} \sum_{i=0}^{N} \sum_{j=0}^{N} \sum_{k=0}^{i} \sum_{s=0}^{j} c_{i j} \frac{(-1)^{s+j+k+1}(s+j) !(i+k) ! \Gamma(\beta-k) \sec \left(\frac{\pi \beta}{2}\right) \sin (\pi(\beta-k))}{2 \pi(j-s) !(s !)^{2}(i-k) ! k ! \ell^{k} \tau^{s}} \\
& \int_{0}^{\ell}\left((-1)^{i} z^{k-\beta}+(\ell-z)^{k-\beta}\right) P_{m}^{\ell}(z) d z \times \int_{0}^{\tau} t^{s} P_{n}^{\tau}(t) d t \\
& -\kappa_{\gamma} \sum_{i=0}^{N} \sum_{j=0}^{N} \sum_{k=0}^{i} \sum_{s=0}^{j} c_{i j} \frac{(-1)^{s+j+k+1}(s+j)(i+k) ! \Gamma(\gamma-k) \sec \left(\frac{\pi \gamma}{2}\right) \sin (\pi(\gamma-k))}{2 \pi(j-s) !(s !)^{2}(i-k) ! k ! \ell^{k} \tau^{s}} \\
& \int_{0}^{\ell}\left((-1)^{i} z^{k-\gamma}+(\ell-z)^{k-\gamma}\right) P_{m}^{\ell}(z) d z \times \int_{0}^{\tau} t^{s} P_{n}^{\tau}(t) d t \\
& -f(z, t) .
\end{aligned}
$$

using Lemma 1, Eq. (4.12) can be written as

$$
\begin{aligned}
& \sum_{i=0}^{N} \sum_{j=0}^{N} \sum_{s=\lceil\alpha\rceil}^{j} \sum_{k=0}^{i} c_{i j} \frac{(-1)^{j+s+k+1}(k+i) !(k !)^{2}((s-\alpha) !)^{2} \Gamma(\alpha-s) \sin (\pi(\alpha-s)) \ell^{k+1} \tau^{\alpha-s}}{\pi(k-m) !(k+N+1) !(s-\alpha-n) !(s-\alpha+n+1) !(i-k) !(k !)^{2}(j-s) ! s ! \ell^{k} \tau^{s}} \\
& -\kappa_{\beta} \sum_{i=0}^{N} \sum_{j=0}^{N} \sum_{k=0}^{i} \sum_{s=0}^{j} c_{i j} \frac{(-1)^{s+j+k+1}(s+j) !(i+k) !(s !)^{2} \Gamma(\beta-k) \sec \left(\frac{\pi \beta}{2}\right) \sin (\pi(\beta-k)) \tau^{s+1}}{2 \pi(j-s) !(s !)^{2}(s-n) !(s+n+1) !(i-k) ! k ! \ell^{k} \tau^{s}} \\
& \times\left((-1)^{i} \frac{\ell^{k-\beta+1}((k-\beta) !)^{2}}{(k-\beta-m) !(k-\beta+N+1) !}+\sum_{k=0}^{N} \frac{(-1)^{k+m}(m+k) ! \Gamma(k+1) \Gamma(k-\beta-1)}{(m-k) !(k !)^{2} \ell^{k} \Gamma(2 k-\beta)}\right) \\
& -\kappa_{\gamma} \sum_{i=0}^{N} \sum_{j=0}^{N} \sum_{k=0}^{i} \sum_{s=0}^{j} c_{i j} \frac{(-1)^{s+j+k+1}(s+j)(s !)^{2}(i+k) ! \Gamma(\gamma-k) \sec \left(\frac{\pi \gamma}{2}\right) \sin (\pi(\gamma-k)) \tau^{s+1}}{2 \pi(s-n) !(s+n+1) !(j-s) !(s !)^{2}(i-k) ! k ! \ell^{k} \tau^{s}} \\
& \times\left((-1)^{i} \frac{\ell^{k-\gamma+1}((k-\gamma) !)^{2}}{(k-\gamma-m) !(k-\gamma+N+1) !}+\sum_{k=0}^{N} \frac{(-1)^{k+m}(m+k) ! \Gamma(k+1) \Gamma(k-\gamma-1)}{(m-k) !(k !)^{2} \ell^{k} \Gamma(2 k-\gamma)}\right) \\
& -f_{m n}=0, \quad 1 \leq m, n \leq N,
\end{aligned}
$$

where

$$
\begin{gathered}
f_{m, n}=\int_{0}^{\tau} \int_{0}^{\ell} f(z, t) \rho_{m n}(z, t) d z d t . \\
\mathcal{B}_{s} y\left(0, t_{j}\right)=\mu_{1}\left(t_{j}\right), \quad 1 \leq j \leq\left\lfloor\frac{N}{2}\right\rfloor, \\
\mathcal{B}_{k} y\left(\ell, t_{j}\right)=\mu_{2}\left(t_{j}\right), \quad 1 \leq j \leq\left\lfloor\frac{N}{2}\right\rfloor+1,
\end{gathered}
$$




$$
y\left(z_{i}, 0\right)=f_{0}\left(z_{i}\right), \quad 1 \leq i \leq\lfloor N\rfloor+\rho
$$

where $\rho= \begin{cases}0, & \mathrm{~N} \text { even; } \\ 1, & \mathrm{~N} \text { odd. }\end{cases}$

Eqs. (4.13)-(4.16) generate an algebraic system of equations of dimension $(N+1)^{2}$ in the vector of unknowns $\mathbf{C}=\left\{c_{i j}\right.$ : $0 \leq i, j \leq N\}$. Hence, the solution can be obtained.

\section{Convergence and error analysis of the proposed expansion}

This section is dedicated to investigating the convergence and error analysis of the suggested double Legendre expansion. In this regard, the following two theorems are stated and proved.

Theorem 4. A function $v(z, t)=f(z) g(t) \in L_{w(z, t)}^{2}(\Omega), w(z, t)=1$ provided with $\left|f^{(3)}(z)\right| \leq M_{1},\left|g^{(3)}(z)\right| \leq M_{2}$ can be expanded as an infinite sum of the basis $\left\{\phi_{i}(z) \psi_{j}(t)\right\}_{0 \leq i, j \leq M}$, and the series converges uniformly to $v(z, t)$. In addition, the expansion coefficients in (3.6) satisfy the following inequality:

$$
\left|c_{i j}\right|<\frac{M_{1} M_{2} \ell^{2} \tau^{2}}{64 i^{2} j^{2}} \quad \forall i, j>1 \text {. }
$$

Proof. From relation (3.7), it follows that

$$
c_{i j}=\frac{(2 i+1)(2 j+1)}{\ell \tau} \int_{0}^{\tau} \int_{0}^{\ell} v(z, t) \phi_{i}(z) \psi_{j}(t) d z d t
$$

and with the aid of Eqs. (3.4) and (3.5), $c_{i j}$ can be written alternatively as

$$
c_{i j}=\frac{(2 i+1)(2 j+1)}{\ell \tau} \int_{0}^{\ell} f(z) P_{\ell, i}(z) d z \int_{0}^{\tau} g(t) \psi_{j}(t) d t .
$$

If the right hand side of Eq. (5.3) is integrated by parts three times, then we get

$$
c_{i j}=\ell^{2} \tau^{2}(2 i+1)(2 j+1) \int_{0}^{\ell} f^{(3)}(z) I_{3, \ell}(z) d z \int_{0}^{\tau} g^{(3)}(t) I_{3, \tau}(t) d t
$$

where

$$
\begin{aligned}
I_{3, b}(z)=\frac{1}{8}[ & \frac{-P_{i-3, b}(z)}{(2 i-3)(2 i-1)(2 i+1)}+\frac{3 P_{i-1, b}(z)}{(2 i-3)(2 i+1)(2 i+3)} \\
& \left.\quad-\frac{3 P_{i+1, b}(z)}{(2 i-1)(2 i+1)(2 i+5)}+\frac{P_{i+3, b}(z)}{(2 i+1)(2 i+3)(2 i+5)}\right] .
\end{aligned}
$$

Now with the aid of the hypothesis $\left|f^{(3)}(z)\right| \leq M_{1},\left|g^{(3)}(t)\right| \leq M_{2}$ along with the inequality $\left|P_{i, b}(z)\right| \leq 1$, we have

$$
\begin{aligned}
\left|c_{i j}\right| & =\left|\ell^{2} \tau^{2}(2 i+1)(2 j+1) \int_{0}^{\ell} f^{(3)}(z) I_{3, \ell}(z) d z \int_{0}^{\tau} g^{\prime \prime \prime}(t) I_{3, \tau}(t) d t\right| \\
& \leq \ell^{2} \tau^{2}(2 i+1)(2 j+1) \int_{0}^{\ell}\left|f^{(3)}(z) I_{3, \ell}(z)\right| d z \int_{0}^{\tau}\left|g^{(3)}(t) I_{3, \tau}(t)\right| d t \\
& <\frac{M_{1} M_{2} \ell^{2} \tau^{2}}{64(2 i-3)(2 i+5)(2 j-3)(2 j+5)} \\
& <\frac{M_{1} M_{2} \ell^{2} \tau^{2}}{64 i^{2} j^{2}}
\end{aligned}
$$

which completes the proof of the theorem. 
Theorem 5. If $y(z, t)$ satisfies the hypothesis of Theorem 4, and if we consider the expansion (3.6), then the truncation error is of $\mathcal{O}\left(N^{-2}\right)$. Explicitly the following error estimate is obtained

$$
\left|y(z, t)-y_{N}(z, t)\right|<\frac{M_{1} M_{2} \ell^{2} \tau^{2}}{64 N^{2}} .
$$

Proof. First, we have

$$
\left|y(z, t)-y_{N}(z, t)\right|=\left|\sum_{i=N+1}^{\infty} \sum_{j=N+1}^{\infty} c_{i j} P_{i}^{\ell}(z) P_{j}^{\tau}(t)\right| .
$$

With the aid of the identity:

$$
\left|P_{k}^{\ell}(z)\right| \leq 1
$$

we get

$$
\left|y(z, t)-y_{N}(z, t)\right|<\sum_{i=N+1}^{\infty} \sum_{j=N+1}^{\infty}\left|c_{i j}\right| .
$$

In virtue of Theorem 4, and if we apply Lemma 2 in [37], then we get

$$
\begin{aligned}
\left|y(z, t)-y_{N}(z, t)\right| & <\frac{M_{1} M_{2} \ell^{2} \tau^{2}}{2^{6}}\left(\int_{N+1}^{\infty} \frac{1}{z^{2}} d z\right)^{2} \\
& =\frac{M_{1} M_{2} \ell^{2} \tau^{2}}{2^{6} N^{2}}
\end{aligned}
$$

which completes the proof of the theorem.

Theorem 6. If the hypothesis of Theorem 4 are satisfied, then we get

$$
\left\|y_{N+1}(z, t)-y_{N}(z, t)\right\|_{\omega}=\mathcal{O}\left(N^{-\frac{3}{2}}\right) .
$$

This means that there exists a generic constant $\epsilon$ such that

$$
\left\|y_{N+1}(z, t)-y_{N}(z, t)\right\|_{\omega} \leq \frac{\epsilon}{N^{\frac{3}{2}}} .
$$

Proof. Based on Theorem 4, following Abd-Elhameed et al. [37], and using $\left\|L_{i}^{\ell}\right\|_{\omega}=\sqrt{\frac{\ell}{2 i+1}},\left\|L_{j}^{\tau}\right\|_{\omega}=\sqrt{\frac{\tau}{2 j+1}}$, the desired result can be obtained.

\section{Numerical results and comparisons}

In this section, some numerical tests supported with some comparisons are given. The two suggested methods in this paper are applied. All the numerical results are obtained via the software "Mathematica 11". By $E_{C}$ and $E_{T}$ we denote, respectively, the maximum pointwise errors resulted from the application of the two methods, namely Shifted Legendre Collocation Method (SLCM) and Shifted Legendre Tau Method (SLTM).

Example 1. Consider the following fractional problem (see, [38]):

$$
\left({ }^{C} D_{t}^{\nu} y\right)(z, t)=\left({ }^{R} D_{z}^{1+\mu} y\right)(z, t)-y(z, t) y_{z}(z, t)+f(z, t), \quad \nu \in(1,2], \quad \mu \in(0,1], \quad(z, t) \in(0,1) \times(0,1),
$$

governed by the conditions

$$
y_{z}(0, t)+y(0, t)=0, \quad y_{z}(1, t)+y(1, t)=0, \quad t \in[0,1],
$$

and

$$
y(z, 0)=z^{2}(1-z)^{2}, \quad y_{t}(z, 0)=-4 z^{2}(1-z)^{2}, \quad z \in[0,1],
$$


where $f(z, t)$ is selected to be compatible with the exact solution of Eq. (6.1) which is $y(z, t)=(1-2 t)^{2} z^{2}(1-z)^{2}$. In [38], Bhrawy considers an approximate solution of $(6.1)$ as $y_{N, M}(z, t)=\sum_{i=0}^{N} \sum_{j}^{M} c_{i, j} P_{L, i}^{(\alpha, \beta)}(z) P_{\tau, j}^{(\alpha . \beta)}(t)$. He obtained an error of order $10^{-17}$, for $N=M=24$. We apply $S L C M$ for $\nu=1.1, \mu=0.8$ and $M=4$. Thanks to the 'RootApproximant' package in Mathematica, we get

$$
\begin{aligned}
& c_{0,0}=\frac{1}{90}, c_{0,1}=0, c_{0,2}=\frac{1}{45}, c_{0,3}=0, c_{0,4}=0, \\
& c_{1,0}=0, c_{1,1}=0, c_{1,2}=0, c_{1,3}=0, c_{1,4}=0, \\
& c_{2,0}=-\frac{1}{63}, c_{2,1}=0, c_{2,2}=-\frac{1}{63}, c_{2,3}=0, c_{2,4}=0, \\
& c_{3,0}=0, c_{3,1}=0, c_{3,2}=0, c_{3,3}=0, c_{3,4}=0 \\
& c_{4,0}=\frac{1}{210}, c_{4,1}=0, c_{4,2}=\frac{1}{105}, c_{4,3}=0, c_{4,4}=0
\end{aligned}
$$

and consequently,

$$
y_{N}(z, t)=z^{2}-4 t z^{2}+4 t^{2} z^{2}-2 z^{3}+8 t z^{3}-8 t^{2} z^{3}+z^{4}-4 t z^{4}+4 t^{2} z^{4}=(1-2 t)^{2} z^{2}(1-z)^{2},
$$

which is the exact solution.

Example 2. Consider the following fractional problem with variable coefficient (see, [36]):

${ }^{C} D_{t}^{\alpha} y(z, t)=a(z){ }^{R} D_{z}^{\beta} y(z, t)-b(z){ }^{R} D_{z}^{\gamma} y(z, t)+f(z, t) \quad 0<\alpha \leq 1, \quad 1<\beta \leq 2, \quad 0<\gamma \leq 1,, \quad(z, t) \in(0,1) \times(0,1)$,

subject to the boundary conditions

$$
y_{z}(0, t)+y(0, t)=0, \quad y_{z}(1, t)+y(1, t)=-t, \quad t \in[0,1]
$$

and the initial condition

$$
y(z, 0)=0, \quad z \in[0,1]
$$

where $a(z)=\Gamma\left(\frac{5}{2}\right) z^{1.5}, b(z)=\Gamma\left(\frac{7}{2}\right) z^{0.5}$ and $f(z, t)$ is selected to be compatible with the exact solution of Eq. (6.2) which is $y(z, t)=z^{2}(1-z) t$. Zhang et al. [36] obtained error of order $10^{-2}$. We apply SLTM for the case corresponding to: $\alpha=0.8, \beta=1.5, \gamma=0.5$ and $M=3$, thanks to the 'RootApproximant' package in Mathematica, we get

$$
\begin{aligned}
& c_{0,0}=\frac{1}{24}, c_{0,1}=\frac{1}{24}, c_{0,2}=0, c_{0,3}=0, \\
& c_{1,0}=\frac{1}{40}, c_{1,1}=\frac{1}{40}, c_{1,2}=0, c_{1,3}=0, \\
& c_{2,0}=-\frac{1}{24}, c_{2,1}=-\frac{1}{24}, c_{2,2}=0, c_{2,3}=0, \\
& c_{3,0}=-\frac{1}{40}, c_{3,1}=-\frac{1}{40}, c_{3,2}=0, c_{3,3}=0,
\end{aligned}
$$

and consequently,

$$
y_{N}(z, t)=t z^{2}-t z^{3}
$$

which is the exact solution.

Example 3. Consider the following fractional problem (see, [5]):

$$
{ }^{R} D_{t}^{\alpha} y(z, t)=B_{1}{ }^{R} D_{|z|}^{\beta_{1}} y(z, t)+B_{2}{ }^{R} D_{|z|}^{\beta_{2}} y(z, t)+f(z, t) \quad \alpha \in(0,1], \quad \beta_{1} \in(0,1], \quad \beta_{2} \in(1,2], \quad(z, t) \in(0,1) \times(0, \tau),(6.3)
$$

subject to the boundary conditions

$$
y_{z}(0, t)+y(0, t)=0, \quad y_{z}(1, t)+y(1, t)=0, \quad t \in[0, \tau]
$$


and the initial condition

$$
y(z, 0)=0, \quad z \in[0,1]
$$

where $f(z, t)$ is selected to be compatible with the exact solution of Eq. (6.3) which is $y(z, t)=\left(t^{\alpha+\beta_{1}}+t^{\beta_{2}}\right) z^{2}(1-z)^{2}$. We apply SLTM and SLCM for the case corresponding to $B_{1}=B_{2}=\frac{1}{2}, \tau=1.5,\left(\beta_{1}, \beta_{2}, \alpha\right)=(0.3,1.5,0.7)$ for different values of $N$. We mention here that the best error obtained by Shen et al. [5] is of order $10^{-4}$. In Table 1, we list the maximum pointwise error.

Table 1: Maximum pointwise error of Example 3.

\begin{tabular}{|c|c|c|c|c|c|}
\hline$N$ & $E_{C}$ & $E_{T}$ & $N$ & $E_{C}$ & $E_{T}$ \\
\hline 7 & $2.27 \times 10^{-2}$ & $6.13 \times 10^{-3}$ & 9 & $5.17 \times 10^{-3}$ & $4.59 \times 10^{-4}$ \\
11 & $5.83 \times 10^{-5}$ & $7.34 \times 10^{-5}$ & 13 & $6.91 \times 10^{-6}$ & $2.61 \times 10^{-6}$ \\
15 & $5.68 \times 10^{-7}$ & $3.28 \times 10^{-7}$ & 17 & $2.24 \times 10^{-8}$ & $9.27 \times 10^{-8}$ \\
\hline
\end{tabular}

Example 4. Consider the following fractional problem:

$$
{ }^{R} D_{t}^{\alpha} y(z, t)=B_{1}{ }^{R} D_{|z|}^{\beta_{1}} y(z, t)+B_{2}{ }^{R} D_{|z|}^{\beta_{2}} y(z, t)+f(z, t) \quad \alpha \in(0,1], \quad \beta_{1} \in(0,1], \quad \beta_{2} \in(1,2], \quad(z, t) \in(0,1) \times(0, \tau),(6.4)
$$

governed by the conditions

$$
y_{z}(0, t)+y(0, t)=0, \quad y_{z}(1, t)+y(1, t)=3 e^{t}, \quad t \in[0, \tau]
$$

and

$$
y(z, 0)=z^{2}, \quad z \in[0,1],
$$

where

$$
f(z, t)=\frac{e^{t}\left(3 \sqrt{\pi} z^{2} \operatorname{erf}(\sqrt{t})-2 \sqrt{2}\left(-2 z^{3 / 2}+2 z \sqrt{1-z}+3 \sqrt{z}+4 \sqrt{1-z}\right)\right)}{3 \sqrt{\pi}},
$$

and $\operatorname{erf}(z)=\frac{2}{\sqrt{\pi}} \int_{0}^{\infty} e^{-z^{2}} d z$ is the error function. The exact solution of Eq. (6.4) is $y(z, t)=z^{2} e^{t}$. We apply SLTM and $S L C M$ for the case corresponding to $B_{1}=B_{2}=1, \tau=1,\left(\beta_{1}, \beta_{2}, \alpha\right)=(0.5,1.5,0.5)$ for different values of $N$. Table 2, lists the resulting maximum pointwise error.

Table 2: Maximum pointwise error of Example 4.

\begin{tabular}{|c|c|c|c|c|c|}
\hline$N$ & $E_{C}$ & $E_{T}$ & $N$ & $E_{C}$ & $E_{T}$ \\
\hline 4 & $5.33 \times 10^{-5}$ & $3.67 \times 10^{-5}$ & 6 & $2.37 \times 10^{-7}$ & $4.82 \times 10^{-7}$ \\
8 & $5.51 \times 10^{-9}$ & $9.27 \times 10^{-9}$ & 10 & $8.30 \times 10^{-11}$ & $5.92 \times 10^{-11}$ \\
12 & $3.82 \times 10^{-13}$ & $4.76 \times 10^{-13}$ & 14 & $2.22 \times 10^{-15}$ & $2.22 \times 10^{-15}$ \\
\hline
\end{tabular}

Example 5. In this case study, we consider the one-dimensional space-time fractional advection-dispersion equation [39]:

$$
\frac{\partial^{\alpha} C}{\partial t^{\alpha}}=D_{1}(z, t) \frac{\partial^{\beta} C}{\partial z^{\beta}}-V_{1}(z, t) \frac{\partial^{\gamma} C}{\partial z^{\gamma}}+V_{2}(z, t) C+G(z, t), \quad(z, t) \in \Omega=(0,1) \times(0,1), \quad 0<\alpha, \gamma<1,1<\beta<2,
$$

governed by the conditions

$$
C(z, 0)=0, \quad z \in(0,1)
$$

$C(1, t)=\frac{t^{2-\alpha}\left(3 \alpha+\frac{2 t}{\Gamma(2-\gamma)}-2 t-9\right)}{\Gamma(4-\alpha)}+\frac{t^{3-2 \alpha}}{\Gamma(4-2 \alpha)}+\frac{t^{2}\left(\frac{(\gamma-2)(\gamma-1) t \Gamma(1-\alpha)}{\Gamma(-\beta-\gamma+3)}+\frac{t \Gamma(3-\gamma)}{\Gamma(3-2 \gamma)}-2 t-9\right)}{6 \Gamma(2-\gamma)}+\frac{1}{6}(t+3)(t+6) t, \quad t \in(0,1)$,

and

$$
C(0, t)=0, \quad t \in(0,1)
$$


The exact nonsmooth solution of Eq.(6.5), see [39] is given by

$$
\begin{aligned}
C(z, t) & =\frac{1}{6}(t+3)(t+6) t z+\frac{z t^{2-\alpha}\left(3 \alpha+\frac{2 t z^{1-\gamma}}{\Gamma(2-\gamma)}-2 t-9\right)}{\Gamma(4-\alpha)}+\frac{z t^{3-2 \alpha}}{\Gamma(4-2 \alpha)} \\
& +\frac{1}{6} t^{2} z^{2-2 \gamma}\left(\frac{z^{\gamma}\left(\frac{(\gamma-2)(\gamma-1) t \Gamma(1-\alpha) z^{1-\beta}}{\Gamma(-\beta-\gamma+3)}-2 t-9\right)}{\Gamma(2-\gamma)}-\frac{(\gamma-2) t z}{\Gamma(3-2 \gamma)}\right) .
\end{aligned}
$$

In this problem, $D_{1}(z, t)=1$ represent the dispersion along positive $z$ direction, $V_{1}(z, t)=z, \quad V_{2}(z, t)=1$ represent the velocity profiles for groundwater, $C(z, t)$ is the contaminant concentration and $G(z, t)$ is additional source or sink term chosen such that the exact solution of (6.5) is given by (6.6). We apply SLTM and SLCM for the case corresponding to $(\alpha, \beta, \gamma)=(0.5,1.5,0.5)$ for different values of $N$. Table 3 , lists the resulting maximum pointwise error.

Table 3: Maximum pointwise error of Example 5.

\begin{tabular}{|c|c|c|c|c|c|}
\hline$N$ & $E_{C}$ & $E_{T}$ & $N$ & $E_{C}$ & $E_{T}$ \\
\hline 4 & $2.51 \times 10^{-1}$ & $4.25 \times 10^{-1}$ & 6 & $5.62 \times 10^{-2}$ & $4.67 \times 10^{-2}$ \\
8 & $2.86 \times 10^{-4}$ & $2.83 \times 10^{-4}$ & 10 & $3.69 \times 10^{-5}$ & $9.31 \times 10^{-5}$ \\
12 & $8.27 \times 10^{-6}$ & $2.84 \times 10^{-6}$ & 14 & $4.57 \times 10^{-7}$ & $1.73 \times 10^{-7}$ \\
\hline
\end{tabular}

\section{Concluding remarks}

In this research article, two robust spectral algorithms have been implemented for handling two different types of space-time fractional advection-dispersion problems. The proposed spectral numerical solutions have been presented in terms of a double Legendre expansion. The tau and collocation methods have been employed to obtain the proposed numerical solutions. The obtained numerical results have indicated that the proposed two methods are accurate and efficient.

\section{Acknowledgments}

The authors would like to thank the referees and the editor for their constructive comments; which helped substantially to improve the manuscript.

\section{References}

[1] F. Liu, P. Zhuang, V. Anh, I. Turner, and K. Burrage. Stability and convergence of the difference methods for the space-time fractional advection-diffusion equation. Appl. Math. Comp., 191(1):12-20, 2007.

[2] P. Zhuang, F. Liu, V. Anh, and I. Turner. Numerical methods for the variable-order fractional advection-diffusion equation with a nonlinear source term. SIAM J. Numer. Anal., 47(3):1760-1781, 2009.

[3] H. Jiang, F. Liu, I. Turner, and K. Burrage. Analytical solutions for the multi-term time-space Caputo-Riesz fractional advection-diffusion equations on a finite domain. J. Math. Anal. Appl., 389(2):1117-1127, 2012.

[4] A.M.A. El-Sayed, S.H. Behiry, and W.E. Raslan. Adomians decomposition method for solving an intermediate fractional advection-dispersion equation. Comput. Math. Appl., 59(5):1759-1765, 2010.

[5] S. Shen, F. Liu, and V. Anh. Numerical approximations and solution techniques for the space-time Riesz-Caputo fractional advection-diffusion equation. Numer. Algorithms, 56(3):383-403, 2011.

[6] Q. Yang, F. Liu, and I. Turner. Numerical methods for fractional partial differential equations with Riesz space fractional derivatives. Appl. Math. Model., 34(1):200-218, 2010. 
[7] K. Wang and H. Wang. A fast characteristic finite difference method for fractional advection-diffusion equations. Adv. water Resour.., 34(7):810-816, 2011.

[8] Q. Huang, G. Huang, and H. Zhan. A finite element solution for the fractional advection-dispersion equation. Adv. Water Resour.., 31(12):1578-1589, 2008.

[9] Y. Zheng, C. Li, and Z. Zhao. A note on the finite element method for the space-fractional advection diffusion equation. Comput. Math. Appl., 59(5):1718-1726, 2010.

[10] A. Yıldırım and H. Koçak. Homotopy perturbation method for solving the space-time fractional advection-dispersion equation. Adv. Water Resour.., 32(12):1711-1716, 2009.

[11] M.M. Meerschaert and C. Tadjeran. Finite difference approximations for fractional advection-dispersion flow equations. J. Comput. Appl. Math., 172(1):65-77, 2004.

[12] F. Huang and F. Liu. The fundamental solution of the space-time fractional advection-dispersion equation. J. Appl. Math. Comp., 18(1):339-350, 2005.

[13] L. Su, W. Wang, and Z. Yang. Finite difference approximations for the fractional advection-diffusion equation. Phys. Lett. A, 373(48):4405-4408, 2009.

[14] C.M. Chen, F. Liu, I. Turner, and V. Anh. A Fourier method for the fractional diffusion equation describing subdiffusion. J. Comput. Phys., 227(2):886-897, 2007.

[15] S.S. Ray and S. Sahoo. Analytical approximate solutions of Riesz fractional diffusion equation and Riesz fractional advection-dispersion equation involving nonlocal space fractional derivatives. Math. Method. Appl. Sci., DOI: 10.1002/mma.3267,2015.

[16] J.C. Parker and U. Kim. An upscaled approach for transport in media with extended tailing due to back-diffusion using analytical and numerical solutions of the advection dispersion equation. Journal of contaminant hydrology, 182:157-172, 2015.

[17] A.H. Bhrawy, M.A. Zaky, and J.A.T. Machado. Efficient Legendre spectral tau algorithm for solving the two-sided space-time caputo fractional advection-dispersion equation. Journal of Vibration and Control, 22(8):2053-2068, 2016.

[18] G. Pang, W. Chen, and Z. Fu. Space-fractional advection-dispersion equations by the kansa method. J. Comput. Phys., 293:280-296, 2015.

[19] Y. Liu and L. Dong. Approximate solutions of multi-order fractional advection-dispersion equation with non-polynomial conditions. Internat. J. Numer. Methods Heat Fluid Flow, 25(1):57-67, 2015.

[20] S. Yeganeh, R. Mokhtari, and J.S. Hesthaven. Space-dependent source determination in a time-fractional diffusion equation using a local discontinuous Galerkin method. Technical report.

[21] S. Chen, F. Liu, X. Jiang, I. Turner, and K. Burrage. Fast finite difference approximation for identifying parameters in a two-dimensional space-fractional nonlocal model with variable diffusivity coefficients. SIAM J. Numer. Anal., 54(2):606-624, 2016.

[22] H Zhang, Fawang Liu, and Vo Anh. Galerkin finite element approximation of symmetric space-fractional partial differential equations. Appl. Math. Comput., 217(6):2534-2545, 2010.

[23] H Zhang, Fawang Liu, Pinghui Zhuang, Ian Turner, and Vo Anh. Numerical analysis of a new space-time variable fractional order advection-dispersion equation. Appl. Math. Comput., 242:541-550, 2014.

[24] H Zhang, F Liu, I Turner, and S Chen. The numerical simulation of the tempered fractional black-scholes equation for european double barrier option. Appl. Math. Model., 40(11):5819-5834, 2016.

[25] A. Napoli and W.M. Abd-Elhameed. An innovative harmonic numbers operational matrix method for solving initial value problems. Calcolo, 54(1):57-76, 2016. 
[26] W.M. Abd-Elhameed. On solving linear and nonlinear sixth-order two point boundary value problems via an elegant harmonic numbers operational matrix of derivatives. Comput. Model. Eng. Sci, 101(3):159-185, 2014.

[27] E.H. Doha and W.M. Abd-Elhameed. Accurate spectral solutions for the parabolic and elliptic partial differential equations by the ultraspherical tau method. J. Comput. Appl. Math., 181(1):24-45, 2005.

[28] E.H. Doha, W.M. Abd-Elhameed, and Y.H. Youssri. Second kind Chebyshev operational matrix algorithm for solving differential equations of Lane-Emden type. New Astron., 23-24:113-117, 2013.

[29] W.M. Abd-Elhameed and Y.H. Youssri. New spectral solutions of multi-term fractional order initial value problems with error analysis. Comp. Model. Eng. Sci, 105:375-398, 2015.

[30] M. Parvizi, M.R. Eslahchi, and M. Dehghan. Numerical solution of fractional advection-diffusion equation with a nonlinear source term. Numer. Algor., 68(3):601-629, 2015.

[31] J. Spanier and K.B. Oldham. An Atlas of Functions. Taylor \& Francis/Hemisphere, 1987.

[32] I. Podlubny. Fractional differential equations: an Introduction to Fractional Derivatives, Fractional Differential Equations, to Methods of Their Solution and Some of Their Applications, volume 198. Academic press, 1998.

[33] E.D. Rainville. Special Functions. New York, 1960.

[34] A.H. Bhrawy and M.A. Zaky. A method based on the Jacobi tau approximation for solving multi-term time-space fractional partial differential equations. J. Comput. Phys., 281:876-895, 2015.

[35] T.H. Koornwinder. On zeilberger's algorithm and its q-analogue. J. Comput. Appl. Math., 48(1-2):91-111, 1993.

[36] Q.H. Zhang, A. Jingguo, and M. Qingwei. Research on generalized space-time fractional convection diffusion equation. J. Chem. Pharm. Res., 6(3):22-26, 2014.

[37] W.M. Abd-Elhameed, E.H. Doha, Y.H. Youssri, and M.A. Bassuony. New Tchebyshev-Galerkin operational matrix method for solving linear and nonlinear hyperbolic telegraph type equations. Numer. Meth. for PDEs, 32(6):1553-1571, 2016.

[38] A.H. Bhrawy. A space-time collocation scheme for modified anomalous subdiffusion and nonlinear superdiffusion equations. Euro. Phys. J. Plus, 131(1):1-20, 2016.

[39] M. K. Singh and A. Chatterjee. Solution of one-dimensional space-and time-fractional advection-dispersion equation by homotopy perturbation method. Acta Geophysica, 65(2):353-361, 2017. 